\title{
AL TRAMONTO DEL GEOCENTRISMO ASTRONOMIA NELLE CINQUECENTINE DELL'ISTITUTO LOMBARDO
}

\author{
Nota del m.e. GIANNANTONIO SACCHI LANDRIANI (*) e \\ ELIO ANTONELLO (**)
}

(Adunanza del 5 marzo 2015)

\begin{abstract}
SUNTO. - Nella presente nota vengono presentate opere edite nel XVI secolo, comunemente dette Cinquecentine, raccolte nella Biblioteca dell'Istituto Lombardo. Si tratta di opere dedicate all'Astronomia concepita nel quadro dell'antica teoria geocentrica, prima della rivoluzione Copernicana. Vengono presentati anche alcuni documenti di quel periodo, relativi a strumenti quali astrolabi e meridiane.
\end{abstract}

$* * *$

ABSTRACT. - In this paper we present some scientific books, edited in XVI century, of the Istituto Lombardo library. These books are devoted to Astronomy conceived in the field of the ancient geocentric theory, just before the heliocentric Copernican revolution. We present also some documents of that period relative to instruments as astrolabes and sundials.

\section{INTRODUZIONE}

Quanto segue è premessa, alla nota su Cinquecentine di Astronomia appartenenti alla nostra biblioteca. ${ }^{1}$

(*) Istituto Lombardo Accademia di Scienze e Lettere, Milano, Italy.

E-mail: giansacchi.landriani@gmail.com

INAF-Osservatorio Astronomico di Brera - Merate, Italy.

E-mail: elio.antonello@brera.inaf.it

1 Nelle didascalie delle figure di frontespizi sono indicate con la simbologia 
Le precedenti comunicazioni su nostre Cinquecentine, hanno toccato temi della Matematica, della Meccanica, delle Scienze Naturali, della Medicina.

Sino ad ora il nostro lavoro ha portato, salvo particolari casi, ad una rassegna bibliografica di opere ricche di contributi storici e figurativi.

E doveroso ricordare la costante collaborazione della nostra Cancelliera e del nostro Bibliotecario e i preziosi contributi di colleghi: Bottani e Rambaldi per un'approfondita comunicazione sulle figure di Tartaglia e Cardano, De Bernardi per le scienze naturali.

Vorremmo giustificare la nostra attenzione a congetture nate in una cultura lontana e scientificamente superata. Si osservi che l'iniziale semplice curiosità di sfogliare i documenti del Cinquecento ha portato a subire il fascino di opere, nate allo svanire di antiche certezze, ma tormentate dallo spirito rinascimentale. Per approfondire dal punto di vista critico la nostra catalogazione sarà necessario l'aiuto dei colleghi umanisti soprattutto per affrontare il latino decadente dei testi denso di neologismi.

In particolare dedicheremo la nostra attenzione al forsennato tramonto del sistema geocentrico nel corso del Cinquecento e accenneremo all'alba della teoria eliocentrica.

L'esplosione editoriale del XVI secolo e la vasta notorietà del problema di svelare il mistero del cielo stellato hanno favorito la notevole diffusione di queste opere, come si può desumere dall'elevato numero di esemplari citati nei cataloghi centrali. ${ }^{2}$ Le evoluzioni concettuali, geometriche e cinematiche di Macrobio (390-430), Gerolamo Fracastoro (1476-1553), Oronzio Fineo (1494-1555), Pietro Apiano (1495-1552), Thyco Brahe (1546-1601) ed altri - ereditate da Aristotele (348-322 a.C.) e Tolomeo (100-175 circa) - costringevano ad una strenua difesa della centralità della terra e del sole visto quale lucente pianeta. Difesa strenua perché sostenuta da un gran numero di scrupolose osservazioni dei movimenti dei pianeti e delle stelle. L'elaborazione dei dati era affi-

[n it/ICCU/xxx...] e [n EDIT 16/xxx...], dove n è il numero di copie indicate nel Catalogo Centrale o rispettivamente nel Catalogo Edit 16 seguite dal rispettivo (xxx) codice identificativo.

2 ICCU Istituto Centrale del Catalogo Unico, EDIT 16 catalogo centrale delle Edizioni del XVI secolo. 
data alla geometria greca e ad elementi di trigonometria, disciplina quest'ultima di antica e misteriosa origine.

Verso la fine del Cinquecento albeggia la teoria eliocentrica con l'emergere della figura di Copernico (1473-1543), ancora tuttavia legato alle orbite circolari e soprattutto timido pubblicista delle sue affermazioni. Timidezza dettata, pare, dal timore di compromettersi all'occhiuta vigilanza del Sant'Uffizio. La Chiesa peraltro non sembra essere intervenuta forse impegnata a difendere la tradizione biblica ma attenta alla nuova teoria.

Sino ad allora gli astronomi erano in sostanza osservatori a occhio nudo del cielo. Il valore dell'esperienza era percepito, ma non pienamente definito. Non esaustivo era l'ausilio della matematica.

È necessario attendere il secolo successivo per vedere Galileo (1564-1642) valersi del primo, seppur elementare, telescopio, e, con Newton, dar vita alla fisica sperimentale, mentre Keplero (1571-1630), coevo di Galileo, definiva, come noto, le orbite ellittiche dei pianeti, e intuiva la gravitazione tra i corpi celesti quale principio del moto non rettilineo. Più tardi Eulero, Lagrange, D’Alembert, Laplace inaugureranno la Fisica-Matematica e la Meccanica Celeste.

\section{Il COSMO}

\subsection{Le sfere omocentriche di Eudosso, Callippo e Aristotele}

Nel periodo che stiamo considerando, il Cinquecento, la concezione del cosmo era ancora basata sulle idee aristoteliche. La Terra era una sfera ferma al centro dell'universo, come si vede in una famosa figura riportata nel libro Cosmographia di Pietro Apiano3 (Figg. 1 e 2). Essa era circondata dagli altri elementi fondamentali e da un sistema di sfere cristalline sulle quali stavano, nell'ordine, la Luna, Mercurio, Venere, il Sole, Marte, Giove e Saturno; infine c'era la sfera delle stelle

3 Petrus Apianus (1495 - 1552) fu un umanista tedesco noto per i lavori di matematica, astronomia e cartografia. Nato Peter Bienewitz, latinizzò il nome (biene = apis, ape). Il suo libro Cosmographicus liber (1524) ebbe grande diffusione come numero di stampe e traduzioni. Apiano fu anche stampatore di opere geografiche e cartografiche di qualità. Ottenne ampi riconoscimenti in vita come uomo di scienza. 
fisse. Il complesso del cosmo era posto in movimento dal primum mobile, oltre il quale stava la sede dei beati e di Dio, ed era considerato perfetto ed immutabile nella sua combinazione di moti circolari uniformi.

La parte del cosmo al di sotto della Luna, o mondo sublunare, era il regno della trasformazione e corruzione dei quattro elementi: il fuoco, l'acqua, l'aria, e infine la terra, ed erano caratterizzati da quattro proprietà: il secco (terra e fuoco), l'umido (aria ed acqua), il freddo (acqua e terra), e il caldo (fuoco e aria), come rappresentato nel libro di Oronzio Fineo ${ }^{4}$ (Figg. 3 e 4). La tendenza naturale del fuoco e dell'aria era salire verso l'alto, mentre quella dell'acqua e della terra era cadere verso il centro della Terra. Invece, il sistema delle sfere celesti cristalline, eterno e incorruttibile, era formato da un elemento, l'etere, che non tendeva né verso il basso né verso l'alto, per cui ogni corpo celeste poteva muoversi solo di moto circolare uniforme.

Il fuoco, diffuso in una sfera posta subito sotto quella della Luna, era una sostanza sottile e trasparente, e quindi non era visibile in condizioni 'normali'. La sfera andava però soggetta ed eventi di corruzione, dai quali scaturivano fenomeni ignei ben visibili come i fulmini, le meteore e i bolidi celesti, e le comete. In altre parole, tale corruzione spiegava gli eventi luminosi nell'atmosfera che non potevano essere di origine terrestre, o comunque fenomeni (come le comete) incompatibili con la perfezione e immutabilità delle sfere superiori. Si potevano generare perfino delle pietre, i meteoriti.

Le comete erano solitamente considerate un presagio di sventura e foriere di sciagure, come le terribili epidemie di peste che avevano segnato la storia dell'umanità. Fin dall'antichità classica l'origine di tali calamità era stata considerata una corruzione o un morbo di origine celeste, come ricordato da Virgilio (Eneide, III, v. 138; Georgiche, III, v. 478). Fino al Seicento gli astronomi furono soprattutto astrologi, e l'astrologia era una branca molto importante del sapere di allora (Fig. 5). Eseguivano calcoli piuttosto complicati, per i quali era necessaria una notevole abilità matematica, al fine di trovare il legame tra gli eventi

4 Oronce Finé (1494-1555), anche Orontius Finaeus o Finnaeus, fu un matematico e cartografo francese. Scrisse molti libri di matematica e astronomia, che, pur contenendo pochi studi originali, contribuirono a diffondere enormemente la conoscenza delle materie scientifiche. Fu anche inventore e costruttore di strumenti. 
incombenti sull'umanità, la posizione degli astri e i fenomeni celesti. Infatti, quanto avveniva sulla Terra era visto come un riflesso di ciò che si poteva leggere un po' in anticipo, e con una certa fatica, in cielo.

Una cinquecentina dell'Istituto Lombardo è intitolata Gnomologia (Fig. 6); il termine, derivato dal greco, indica una raccolta di sentenze. Il libro consiste in citazioni tratte da vari dialoghi di Platone. Nel Timeo, Platone aveva esposto le proprie idee sulla formazione del cosmo, sulla storia della sua evoluzione e degli uomini che lo abitano, sull'importanza del numero e dell'armonia cosmica. Per esempio, secondo Platone era stato proprio grazie all'osservazione del cielo che l'umanità aveva imparato a contare (e così erano nate l'aritmetica e la matematica) e a riflettere (e così era nata la filosofia): "Ora, le osservazioni del giorno e della notte, dei mesi e dei periodi degli anni, degli equinozi e dei solstizi hanno procurato il numero, e hanno fornito la riflessione sul tempo e la ricerca sulla natura dell'universo: da queste cose abbiamo ottenuto il genere della filosofia" (Platone, Timeo [47 a, b]). Tali considerazioni erano ulteriormente ribadite nel dialogo Epinomide (opera di un allievo): "Come abbiamo imparato a contare ... Fra le realtà che il dio ci mostra, c'è qualcosa di più bello del vedere sorgere il sole e poi prendere parte allo spettacolo della notte, che offre una visione del tutto differente? ... E poiché il cielo si rinnova ciclicamente, senza cessare di far seguire infinite notti a infiniti giorni, non smetterà di insegnare agli uomini l'uno e il due, prima che anche l'uomo più tardo non abbia imparato a contare in modo decente ..." (Epinomide, [978 b, c, d]).

Le concezioni di Platone erano state in parte criticate da Aristotele, ma altri autori, come i neoplatonici, ne avevano mantenuto l'impostazio-

5 Lo stesso Galileo fu autore di oroscopi, o 'temi natali'. Luca Gaurico (14751558) può essere considerato un tipico rappresentante di questa categoria; scrisse numerosi 'temi natali', facendone un uso piuttosto disinvolto, per non dire sfacciato, ma pubblicò anche diversi lavori teorici, sia come autore sia come curatore. Praticare l'astrologia era un modo per avere un guadagno sicuro, facendo leva (ieri come oggi) sulla psicologia umana e sulla sua attitudine di fronte all'incertezza del futuro, in particolare di coloro che detenevano il potere politico. Comunque, nel 1586 il papa Sisto V emanò la bolla Coeli et terrae con la quale venivano condannate l'astrologia giudiziale (o giudiziaria) e la divinazione, che esprimevano giudizi sul futuro dell'uomo, perché ciò andava contro il suo libero arbitrio, e perché solo Dio conosceva il futuro. Era considerata accettabile l'astrologia naturale, che studiava gli eventi celesti con finalità pratiche, per esempio in campo medico. 
ne filosofica. E' il caso di Macrobio ${ }^{6}$ (Figg. 7 e 8) e del suo commento piuttosto esteso al Somnium Scipionis di Cicerone; il libro ebbe una certa fortuna nel Medioevo, vista la diffusione di temi platonici e neoplatonici nel pensiero medievale. Nel De Re Publica, Cicerone aveva descritto la visione avuta in sogno da Scipione Emiliano prima di distruggere Cartagine. L'Emiliano raccontava di aver incontrato in cielo il nonno adottivo Scipione l'Africano. Il nonno gli aveva mostrato la sede dei beati, la Via Lattea (il grande cerchio che incrocia quello dello zodiaco tra Toro e Gemelli da una parte, e Scorpione e Sagittario dall'altra), e la struttura del cosmo. L'Emiliano era particolarmente colpito dalla Terra, piccolissima vista dalle stelle; Macrobio, nel Libro II, aveva commentato in dettaglio le zone climatiche terrestri, con due fasce temperate abitabili molto strette (cioè, aventi piccola estensione lungo i meridiani) rispetto alle due zone polari gelide, e rispetto alla fascia torrida equatoriale molto estesa. ${ }^{7}$ E' da notare che, alla fine dell'Impero, pochi autori cristiani erano convinti che la Terra fosse piatta; c'era però discussione sull'esistenza o meno degli abitanti degli antipodi, e già Aristotele aveva escluso l'abitabilità dell'emisfero australe. A proposito della forma della Terra, ricordiamo che Apiano nella Cosmographia aveva segnalato l'ombra della Terra proiettata sulla Luna durante un'eclissi del nostro satellite quale dimostrazione diretta della sua sfericità; infatti, la forma dell'ombra non era compatibile, per esempio, con un poligono (triangolo, quadrato, esagono).

Com'era fatto il sistema delle sfere omocentriche? Il modello, elaborato da Eudosso e modificato poi da Callippo, nel IV sec. a.C., era costituito da 33 sfere, tre o quattro per ogni pianeta, e ognuna era in moto uniforme attorno a un proprio asse, diverso da quello delle altre. Il sistema era molto complicato perché doveva spiegare le irregolarità del moto

6 Ambrogio Teodosio Macrobio (Ambrosius Theodosius Macrobius, circa 390430) fu un filosofo e funzionario dell'Impero. Oltre ai Commentarii in Somnium Scipionis, scrisse il dialogo Saturnalia e un'opera di grammatica sul verbo greco e latino.

7 La concezione del cosmo all'epoca dell'Impero era stata discussa in dettaglio per esempio da Plinio (I sec. d.C.). In particolare, egli scrisse della divisione della Terra e del cielo circostante "in cinque parti, chiamate zone", dove "un freddo ostile e un gelo eterno opprimono tutte le terre sottoposte alle due parti più esterne, verso l'uno e l'altro polo: il nostro, che è chiamato Settentrione, e l'altro, opposto, che ha nome Australe ... Mentre la fascia mediana, dove passa l'orbita del Sole, bruciata dalle fiamme e surriscaldata dalla vicinanza della fonte di calore, è torrida. Solo le due circostanti, cioè quelle comprese fra la zona surriscaldata e le zone gelate, sono temperate e non comunicano fra loro per l'ardore del cielo" (Plinio, Storia Naturale, I, 68). 
planetario, in particolare quello apparente retrogrado. Osservando un pianeta esterno del Sistema Solare, come Marte, quando la Terra si trova tra il pianeta e il Sole (in opposizione), e seguendone il movimento per alcuni mesi, lo vediamo spostarsi, lentamente, verso est rispetto alle stelle, finché rallenta e prende a muoversi verso ovest, e quindi dopo un paio di mesi riprende il lento moto verso est. Questo fenomeno è facilmente spiegabile nel sistema eliocentrico come un effetto di prospettiva legato alle diverse velocità orbitali della Terra e di Marte, ma diventa complicato se lo si vuole giustificare nei sistemi geocentrici. A Schiaparelli è generalmente riconosciuto dagli storici dell'astronomia il merito di aver spiegato in modo chiaro il principio di funzionamento del sistema delle sfere omocentriche; tra l'altro, illustrò la sua idea proprio all'Istituto Lombardo durante un'adunanza nel 1874 (Schiaparelli, 1875; Dreyer, 1970).

Aristotele (Fig. 9) aveva aumentato il numero delle sfere portandolo a 54, e aveva esposto le sue idee sul cosmo, che abbiamo ricordato all'inizio, nella Fisica e nel De caelo. Nel Cinquecento, Fracastoro ${ }^{8}$ (Fig. 10) introdusse altre sfere fino a un totale addirittura di 77. Tuttavia, nel sistema che stiamo considerando, un pianeta si trova sempre alla stessa distanza dalla Terra, e quindi il modello non era in grado di spiegare la variazione della luminosità dell'astro lungo la sua orbita, dovuta proprio alla variazione della sua distanza dalla Terra. Per Fracastoro, per esempio, le variazioni di luminosità dipendevano da una diversa densità nelle varie parti del cielo (Delambre, 1819, p. 387).

\subsection{Il sistema tolemaico}

Durante il Rinascimento era divenuto familiare anche il modello tolemaico, pur esso complicato, con epicicli e deferenti in moto circo-

8 Il veronese Girolamo Fracastoro (1483-1553) studiò all'Università di Padova all'inizio del Cinquecento, quando vi si trovava come studente anche Copernico. Visse a Verona, dedicandosi, con un'attività poliedrica, alla medicina, all'astronomia, alla filosofia e alla poesia. Pubblicò gli studi di astronomia, Homocentrica, nel 1538. "Si può sperare che Fracastoro comprendesse il suo sistema in tutti i particolari, ma certo non aveva il dono di dare ai suoi lettori una chiara idea di ogni dettaglio dell'ingombrante meccanismo ... L'oscurità della descrizione può essere stata tra le cause del totale insuccesso del libro" (Dreyer, p. 271). La cinquecentina contenente l'Homocentrica (Fig. 10) è l'Opera Omnia di Fracastoro pubblicata, in varie edizioni, dopo la sua morte; la sua diffusione sarebbe quindi da ascriversi forse più agli altri testi, come quelli di medicina, inclusi nel libro. 
lare uniforme. Un pianeta si muove lungo un cerchio (epiciclo) il cui centro si sposta lungo un altro cerchio (deferente); in particolare, il centro geometrico del deferente non era la Terra, bensì un punto simmetrico tra la Terra e l'equante. A sua volta, l'equante era definito come punto rispetto al quale la rivoluzione lungo il deferente avveniva con velocità angolare costante. In questo modo si salvava il principio dei moti circolari uniformi che doveva stare alla base del moto (all'apparenza irregolare) di rivoluzione dei pianeti. Quello di Tolomeo (II sec. d.C.) era però essenzialmente un modello matematico, mentre quello 'vero' era l'aristotelico. Il sistema degli epicicli spiegava la variazione di distanza, ma era incompatibile con il sistema delle sfere cristalline.

Nel Cinquecento, il polacco Copernico (1473-1543) aveva costruito il suo modello eliocentrico secondo l'approccio tolemaico, cioè complicato dalla presenza di molti epicicli e deferenti in moto circolare uniforme, e anch'esso era considerato per lo più un modello matematico. Il suo libro De Revolutionibus venne pubblicato nel 1543. Anni dopo, il danese Tycho Brahe (1546-1601), non soddisfatto del modello copernicano, pensò ancora alla Terra posta al centro dell'Universo, ma con Mercurio e Venere in orbita intorno al Sole, il quale a sua volta era in orbita intorno alla Terra; anche i pianeti esterni, Marte, Giove e Saturno, avevano il Sole come centro della loro orbita. Brahe lasciò in eredità a Keplero (15711630) una grande quantità di osservazioni accurate, compiute nei suoi Osservatori con l'aiuto di vari collaboratori, perché sviluppasse questa sua idea che meglio rappresentava i dati osservativi allora disponibili. Però Keplero capì che un pianeta come Marte, così 'difficile' nei sistemi geocentrici, diventava un caso semplice se pensato su un'orbita ellittica intorno al Sole, dando così ragione al principio copernicano, ma allo stesso tempo semplificando parecchio il modello. Keplero pubblicò i primi risultati nel 1609, lo stesso anno in cui Galileo (1564-1642) puntava per la prima volta il telescopio verso il cielo.

Come affermato da Dreyer, quello "dal 1543 al 1609 fu un periodo di transizione, in attesa che il sistema di Copernico venisse liberato dai suoi elementi caduchi e rafforzato da Keplero" (Dreyer, p. 316). Ci furono uomini di scienza che accettarono il principio copernicano, e altri che ammettevano il moto diurno della Terra ma non quello orbitale. "Per molti anni dopo la pubblicazione dell'opera di Copernico, l'astronomia non fece alcun progresso; un'opinione valeva l'altra" (Dreyer, p. 328), e "benché l'astronomia e l'astrologia attraessero un gran numero di studiosi ..., la teologia veniva ancora considerata l'alfa 
e l'omega. E la teologia era venuta a significare la più letterale accettazione di ogni parola della Scrittura; per i protestanti di necessità, poiché essi avevano negato l'autorità dei Papi e dei Concili; per i cattolici romani a causa del desiderio di definire con maggior precisione le loro dottrine ... In questo momento veniva a cadere ogni possibilità di parlare di Rinascimento cristiano" in Europa (Dreyer, pp. 322-323).

\section{STRUMENTI E TECNICHE}

Fino all'avvento del telescopio di Galileo, le osservazioni astronomiche erano state effettuate ad occhio nudo con l'ausilio di alcuni strumenti specifici per la misura della posizione degli oggetti celesti, e di altri che agevolassero l'osservazione. Giacomo Leopardi, per esempio, nelle Operette Morali notava che Copernico guardava in cielo "per mezzo d'un cannoncello di carta; perché non erano ancora inventati i cannocchiali" (Il Copernico, Scena Seconda); ci sono illustrazioni in alcuni codici medioevali che mostrano tale operazione. Il tubo aveva lo scopo di migliorare la visibilità di stelle e pianeti, perché eliminava molta parte della luce parassita che colpiva la pupilla (cioè, la luce proveniente da altri oggetti celesti o dall'ambiente circostante, oppure diffusa dal cielo).

\subsection{Planisferi, astrolabi, torqueto}

Il planisfero è la rappresentazione proiettata su un piano della sfera terrestre (nel caso del planisfero geografico), o della sfera celeste (nel caso degli astrolabi). Ci sono vari libri dedicati a tali strumenti, come quelli di Gallucci ${ }^{9}$ (Fig. 11) e Guidobaldo Del Monte ${ }^{10}$. Il planisfero geografico aveva una struttura a griglia con linee radiali che rappresentavano i meridiani, e archi concentrici che rappresentavano i

9 Giovanni Paolo Gallucci (1538-1621) visse a Venezia, dove si dedicò in particolare all'insegnamento privato dei giovani nobili, pubblicò traduzioni in volgare di testi scientifici, di storia naturale e di arte militare, e un libro divulgativo dalla ricca iconografia per spiegare i fenomeni celesti anche ai non esperti. Scrisse vari libri sulla costruzione e uso di orologi e strumenti astronomici.

10 Guidobaldo Del Monte (1545-1607) fu un matematico, filosofo ed astronomo; visse per lo più a Pesaro; fu amico e corrispondente di Galileo. Scrisse libri di meccanica (Mechanicorum liber), ed è ricordato per il rigoroso controllo sperimentale cui 
paralleli. Al centro era imperniato un indice che serviva a determinare le distanze tra la città di riferimento e i vari luoghi sulla Terra, su scala molto grande (Figg. 12, 13 e 14).

Con il termine astrolabio (in greco astrolábon, che prende/comprende le stelle) sono stati chiamati strumenti diversi tra loro, adibiti a rilevare le posizioni degli astri oppure utilizzati nel calcolo astronomico: astrolabio piano, astrolabio universale e di Rojas, astrolabio nautico. Nato in epoca ellenistica, fu sviluppato nell'Islam in diverse varianti, e le traduzioni di testi arabi ne diffusero la conoscenza in Europa, in epoca medioevale; solo nel XVII secolo, con la costruzione di orologi meccanici accurati e l'elaborazione di nuovi metodi di calcolo, lo strumento divenne obsoleto. Ignazio Danti ${ }^{11}$ descrisse la costruzione e l'uso di tali strumenti (Fig. 15). Un astrolabio piano (Figg. 16, 17) si riferisce alle coordinate altazimutali dell'osservatore, cioè la posizione di un oggetto celeste è definita rispetto all'orizzonte del luogo e allo zenit. Lo strumento era valido quindi solo per la latitudine del luogo. Si cercò di superare l'inconveniente con gli astrolabi universali, che erano applicabili alle altre latitudini, ma risultarono poco pratici. Ricordiamo infine che l'astrolabio nautico, diverso dall'astrolabio piano, era usato per misurare la distanza zenitale del Sole o di una stella, in particolare la Polare, nel momento del transito al meridiano, al fine di determinare la latitudine toccata dalla nave (Museo Galileo, 2015).

Il torqueto astronomico (Fig. 18) era uno strumento piuttosto complesso, prodotto in Europa dalla fine del XIII secolo, e poteva essere usato per misurare un oggetto celeste nei tre sistemi di coordinate altazimutale, equatoriale ed eclittico. Nel caso equatoriale le coordinate sono riferite al piano dell'equatore terrestre e alla direzione del Polo Nord celeste; nel caso eclittico sono riferite al piano dell'orbita terrestre (eclittica) e alla direzione del Polo dell'orbita. Lo strumento era utile anche come calcolatore analogico, per passare da uno dei sistemi di coordinate all'altro.

sottoponeva le sue conclusioni. Nel Planisphaeriorum universalium theorica tentava un miglioramento dei planisferi universali.

11 Ignazio o Egnatio Danti (1536-1586), domenicano, dal 1583 vescovo di Alatri, è stato un matematico, astronomo e cosmografo. Insegnò matematica all'Università di Bologna, dove costruì la prima meridiana di San Petronio. A Roma fece parte della commissione per la riforma del calendario voluta da Gregorio XIII, e disegnò i cartoni per gli affreschi delle carte geografiche in Vaticano. 


\subsection{Orologi}

Fin dall'antichità, la specializzazione dell'attività umana e la crescente complessità della società avevano resa necessaria la suddivisione del tempo giornaliero in unità facilmente misurabili. Questo era possibile con la meridiana, osservando la posizione dell'ombra dello gnomone illuminato dal Sole, come illustrato da Giovanni Battista Vimercati (Fig. 19). La lunghezza dell'ombra varia in funzione della data e del momento della giornata. Per esempio, nel caso della meridiana orizzontale, alle latitudini intermedie, lontano dall'equatore e dai poli, la lunghezza passa da un valore minimo al solstizio d'estate a un valore massimo al solstizio d'inverno, e, durante la giornata, l'ombra compie una curva raggiungendo il valore più piccolo al momento del mezzogiorno locale; agli equinozi, l'estremo dell'ombra percorre una linea retta (Fig. 20). ${ }^{12}$

Per determinare le ore notturne si misurava la posizione relativa delle stelle usando il notturlabio, o notturnale, od orologio notturno. La posizione delle stelle, infatti, dipende dalla data e dal momento di osservazione durante la notte. In particolare, venivano puntate le stelle principali dell'Orsa Maggiore in riferimento alla Polare. L'instrumentum syderale di Pietro Apiano è una delle prime descrizioni di tale "orologio" (Fig. 21).

\subsection{Tavole astronomiche}

In epoca alto-medievale c'era stato un regresso delle scienze in Europa; l'astronomia era stata coltivata da cultori islamici in Asia, e nei secoli XI e XII alcuni dei principali astronomi si trovavano nella Spagna musulmana. Il re Alfonso X di Castiglia, detto il Saggio (1252-

12 Con questa meridiana la durata di un'ora è sempre la stessa. Per molti secoli però si era continuato ad usare il sistema antico di dividere il periodo di luce, dall'alba al tramonto, in dodici ore tra loro uguali, e la notte altrettanto. Di conseguenza, le ore diurne estive erano lunghe e quelle invernali brevi: per esempio, alla latitudine di Milano, al solstizio d'estate un'ora diurna durava circa 76 minuti, e al solstizio d'inverno circa 43 minuti. Nel caso di tale applicazione, le rette sul piano della meridiana hanno un andamento diverso. Aggiungiamo inoltre che, nel Ducato di Milano e in altre località italiane, fino al XVIII secolo si mantenne l'antica consuetudine di iniziare il giorno (e quindi il conto delle ore) al tramonto del Sole. Per una discussione dettagliata della proposta di introdurre l'attuale sistema ('francese') invece dell'antico romano anche a Milano, si veda il saggio storico-erudito (ma anche gustoso) di Longo (1766, p. 444) su Il Caffè. 
1284) è ricordato in particolare per il grande interesse verso l'astronomia e la cultura. ${ }^{13}$ Egli chiamò alla sua corte, a Toledo, astronomi ebrei, cristiani e musulmani, per lavorare alle sue dipendenze in vista della preparazione di nuove tavole astronomiche, poi chiamate Tavole alfonsine (Fig. 22), elaborate con lo scopo di migliorare e aggiornare quelle tolemaiche contenute nell' Almagesto. Il lavoro terminò nel 1252, l'anno in cui Alfonso divenne re. Le Tavole fornivano, tra l'altro, le posizioni del Sole, dei pianeti e delle stelle fisse, congiunzioni astronomiche, caratteristiche di eclissi, durata di ere storiche (regni), calendario e tabelle di conversione; il meridiano di riferimento era quello di Toledo, e i calcoli erano riferiti all'anno 1256. I principi teorici su cui il lavoro era stato impostato erano sempre quelli di Tolomeo; inoltre, era stata considerata una precessione media degli equinozi che era circa la metà del reale, con in più la complicazione della 'trepidazione', una supposta oscillazione (in realtà inesistente) degli equinozi stessi (Delambre, 1819, p. 248). Le Tavole godettero di grande fama per tre secoli; furono diffuse dapprima come codici manoscritti, e vennero stampate per la prima volta nel 1483. Erano le migliori tavole allora esistenti, per cui vennero continuamente usate, riviste e integrate sia dagli astronomi sia dai navigatori (era ormai l'epoca delle scoperte geografiche), in base alle nuove osservazioni, ed erano usate anche per fini astrologici.

L'edizione accresciuta del 1545 stampata a Parigi (a cura di P. Hamellius), rispetto a quella di Venezia del 1492 (a cura di J.L. Santritter), include, tra l'altro, alcune tavole di Giovanni Bianchini e di Luca Gaurico. ${ }^{14}$ Contiene anche una breve discussione della data dell'equinozio di primavera, del conseguente problema della data di Pasqua, e su come correggere i difetti del calendario giuliano. Infatti, tale calendario, in uso fin dai tempi di Giulio Cesare, aveva accumulato progressivamente una discrepanza temporale di una decina di giorni tra la data 'ufficiale' dell'equinozio di primavera (21 marzo) e l'evento astronomico reale dell'equinozio nel XVI secolo (circa 10 marzo). ${ }^{15}$ Ciò costituiva

13 Alfonso X patrocinò la composizione di un'estesa letteratura, che segnò l'inizio della prosa castigliana, in vari campi del sapere (poetico, giuridico, storico e scientifico), contribuendovi anche in prima persona, e supervisionando il lavoro degli autori letterati cristiani, ebrei e musulmani.

14 Giovanni Bianchini (1410-1469) fu matematico e astronomo dell'Università di Ferrara. Per Gaurico, v. nota 5.

15 Un calendario assume per la durata di un anno un numero intero di giorni, 
un problema per il calcolo della data di Pasqua, perché essa dovrebbe cadere la prima domenica dopo il plenilunio della primavera astronomica, cioè il primo plenilunio dopo l'equinozio vero. Il papa Gregorio XIII istituì allora una commissione per riformare il calendario, e nel 1582, con la bolla Inter gravissimas, promulgò il nuovo sistema, in uso tutt'oggi. Al 1582 furono tolti 10 giorni, e il giorno successivo a giovedì 4 ottobre fu venerdì 15 ottobre, e venne introdotta una correzione nella determinazione degli anni bisestili. ${ }^{16}$

\subsection{Trattati di ottica}

Il libro Opticae Thesaurus (Fig. 23) contiene tre trattati: a) uno sull'ottica e su altri argomenti correlati, composto da Ibn al-Haytham (autore dell'XI secolo noto come Alhazen), dove sono discusse la percezione visiva, la teoria della luce e dei colori, la riflessione e la rifrazione; $b$ ) un trattato in cui è discusso il crepuscolo; $c$ ) un testo contenente i lavori di ottica di Witelo ${ }^{17}$ (Vitellonis Thuringopoloni opticae libri decem). Il lavoro di quest'ultimo autore (Fig. 24) tratta in particolare della prospettiva, e fu utilizzato da artisti rinascimentali come il Ghiberti.

mentre l'anno tropico medio, cioè il tempo perché il Sole compia un'orbita apparente uniforme di 360 gradi esatti, dura 365,24218 giorni. Già nell'antichità con la riforma giuliana avevano cercato di superare il problema introducendo l'anno bisestile di 366 giorni alternato a tre anni comuni di 365 giorni; questo però implica una durata media dell'anno di 365,25 giorni, e la differenza rispetto all'anno tropico (come era stimato all'epoca) spiega l'accumulo avvenuto nell'arco di sedici secoli.

16 Sono solitamente bisestili gli anni divisibili per 4, come, per esempio, il 1932. Nel sistema giuliano la cadenza di anni bisestili era leggermente in eccesso, e la soluzione era quindi ridurla togliendo qualcuno degli anni bisestili. Nella cinquecentina del 1574 si proponeva di non considerare bisestili, ma comuni, gli anni 1660, 1796, 1932, 2068, ecc. (separati tra loro di 136 anni). La riforma gregoriana introdusse la regola mnemonica che non erano bisestili gli anni secolari non divisibili per 400, per esempio 1700, 1800, 1900; in questo caso la separazione media tra gli 'ex'-bisestili è 133 anni. Resta comunque ancora una piccola discrepanza, perché l'anno medio gregoriano risulta così di 365,2425 giorni; gli effetti però si vedono su tempi scala molto lunghi, con l'accumulo di un giorno ogni 3000 anni circa. Eventualmente, sarà argomento di discussione nei prossimi millenni considerare comune qualche altro anno bisestile.

17 Erazmus Ciolek Witelo (circa 1230 - 1280) fu un matematico, fisico, filosofo e teologo, nato in Slesia e vissuto in Turingia. Il suo lavoro Perspectiva si basava su quello di Alhazen. 
Ci sembra opportuno concludere questa parte riallacciandoci a Keplero. L'astronomo nel 1604 aveva scritto infatti un supplemento ai libri di Witelo, Ad Vitellionem paralipomena, quibus Astronomiae pars optica traditur (una copia del libro è conservata all'Osservatorio Astronomico di Brera), dove aveva discusso, oltre alla percezione visiva e alla teoria della luce, anche argomenti prettamente astronomici. Keplero riprenderà tali argomenti nell'Astronomia Nova, il testo del 1609 che, insieme alle osservazioni al telescopio di Galileo, contribuirà in modo determinante alla rivoluzione scientifica del XVII secolo.

\section{OSSERVAZIONI GENERALI}

È sorprendente l'atteggiamento degli studiosi del Cinquecento, privi di strumenti se non del proprio occhio e di qualche astrolabio, intenti a rappresentare l'universo e a proporre diversi modelli del sistema planetario geocentrico. Erano anche Astrologi, il che proietta un'ombra sulla loro figura di cultori della scienza nell'attuale accezione. Ma erano dotati di splendida immaginazione.

Soprattutto volevano svelare il sistema dell'universo. Le loro congetture non furono completamente dimenticate e in parte suggerirono la realizzazione di capacità operative d'almeno quattro secoli dopo. Oggi sappiamo navigare nel nostro sistema planetario e osservare lontane galassie perché grandi sognatori di un tempo remoto hanno guardato e amato le stelle.

Il che conforta coloro che ancora oggi credono nella necessità di coltivare le scienze prive di riscontri immediati in termini di risultati concreti e di profitti economici. E credo sia questo il fascino che le Cinquecentine trasmettono a noi più eruditi ma non più sapienti di loro. Anche i perdenti strenui difensori di quanto sarebbe stato negato nel volgere di pochi decenni, meritano il nostro profondo rispetto. Ricordano che la ricerca ha in sé un grande valore che non implica necessariamente il trovare. 


\section{BIBLIOGRAFIA}

Copernicus N., ex Officina Henricpetrina 1566, De revolutionibus orbium coelestium libri VI... per M. Georgium Ioachimum Rheticum ad Ioan. Schonerum scripta. Osservatorio Astronomico di Brera in Milano, n. 160.36 in folio.

Delambre M., 1819, Histoire del l'astronomie du Moyen Age, Paris: Courcier.

Dizionario Biografico degli Italiani, http://www.treccani.it/enciclopedia/, alle voci: Luca Gaurico, Girolamo Fracastoro, Giovanni Paolo Gallucci, Guidobaldo del Monte, Ignazio Danti.

Dreyer J.L.E., 1970, Storia dell'astronomia da Talete a Keplero, Milano: G. Feltrinelli Editore.

EDIT 16, Catalogo centrale di Edizioni del XVI secolo.

Keplero J., 1604, Ad Vitellionem paralipomena, quibus Astronomiae pars optica traditur, Francofurti, apud Claudium Marnium \& haeredes Ioannis Aubrii. Osservatorio Astronomico di Brera n. 170.106 -in quarto ICCU, Istituto Centrale del Catalogo Unico.

Leopardi G., 1835, Operette Morali, Il Copernico.

Longo A., 1766, Dissertazione sugli orologi, in Il Caffè o sia brevi e varj discorsi già distribuiti in fogli periodici, Tomo Primo, Venezia: Pizzolato.

Museo Galileo, http://catalogo.museogalileo.it (gennaio 2015).

Schiaparelli G.V., 1875, Le sfere omocentriche di Eudosso, di Callippo e di Aristotele. Memoria di G.V. Schiaparelli letta al Reale Istituto Lombardo di Scienze e Lettere nell'adunanza del 26 novembre 1874, Milano: Ulrico Hoepli, Pubblicazioni del Reale Osservatorio di Brera in Milano, n. 9.

Platone, Tutte le opere, a cura di E.V. Maltese, Roma, Newton Compton, 1995.

Plinio, Storia Naturale, I, Cosmologia e geografia, Libri 1-6, trad. di A. Barchiesi, Giulio Einaudi Editore. 


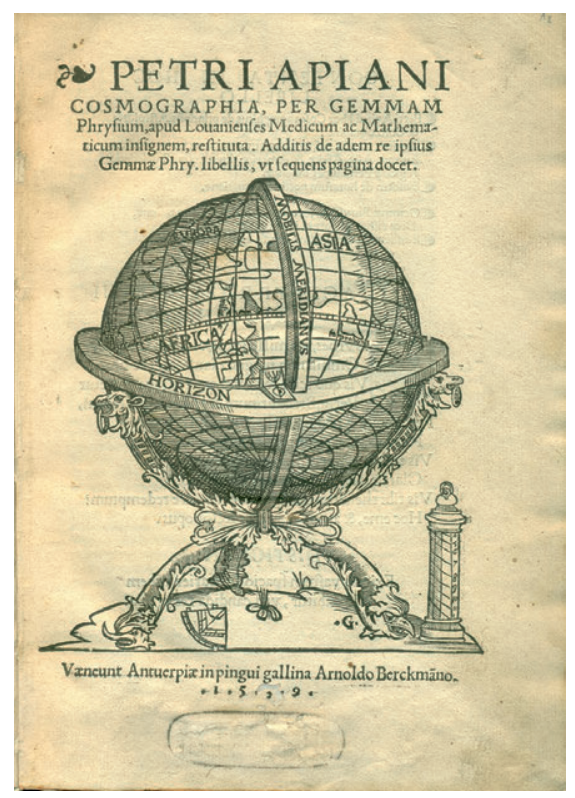

Fig. 1 - Frontespizio.

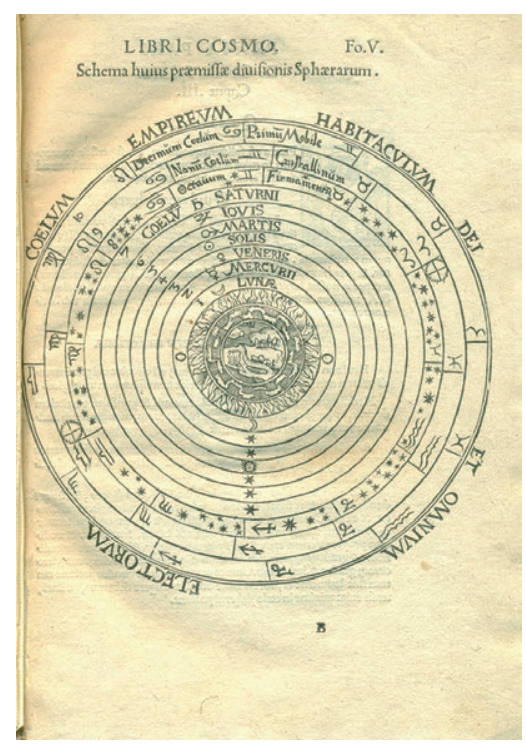

Fig. 2 - Cosmo

PETRI APIANI (1495-1552) COSMOGRAPHIA-Antuerpiae (1539?) 1524 Astronomo, matematico, cartografo sassone $h .23 \mathrm{~cm}$. 


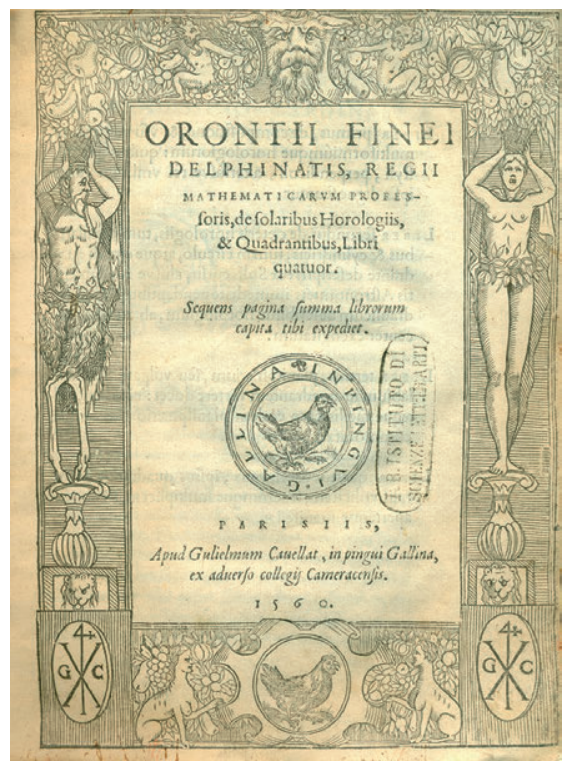

Fig. 3 - ORONZIO FINEO (Briançon 1494-Parigi 1555) De solaribus Horologiis E Quadrantibus, Libri quatuor PARISIIS h.27 cm.

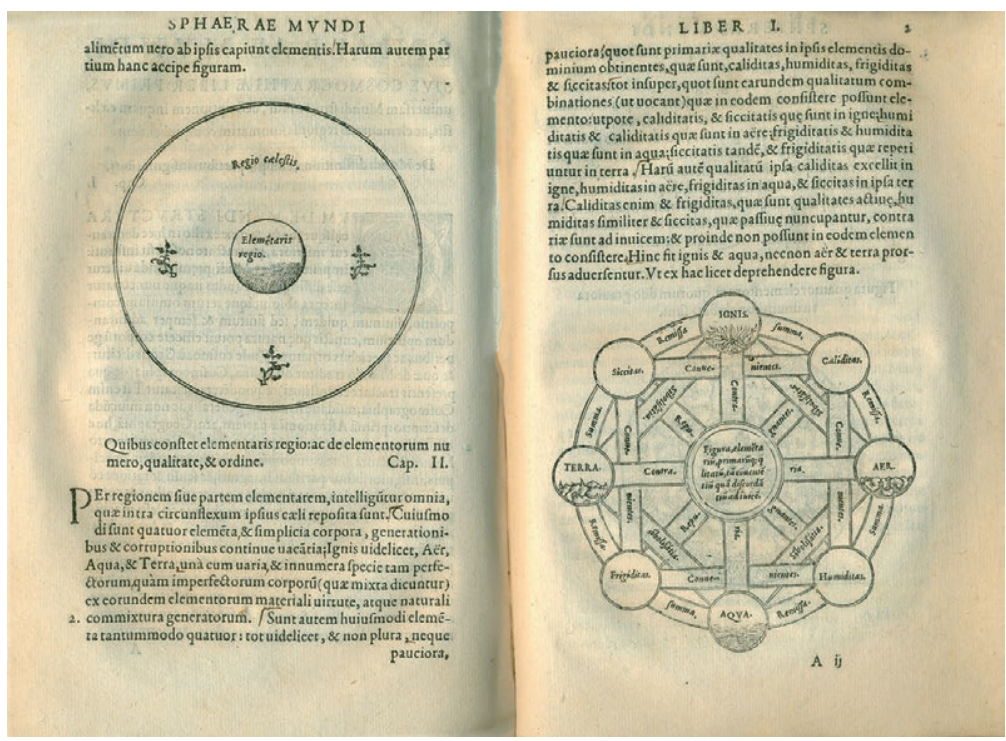

Fig. 4 - O. FINEO IDEM Sphaerae mundi Liber I ELEMENTI. 


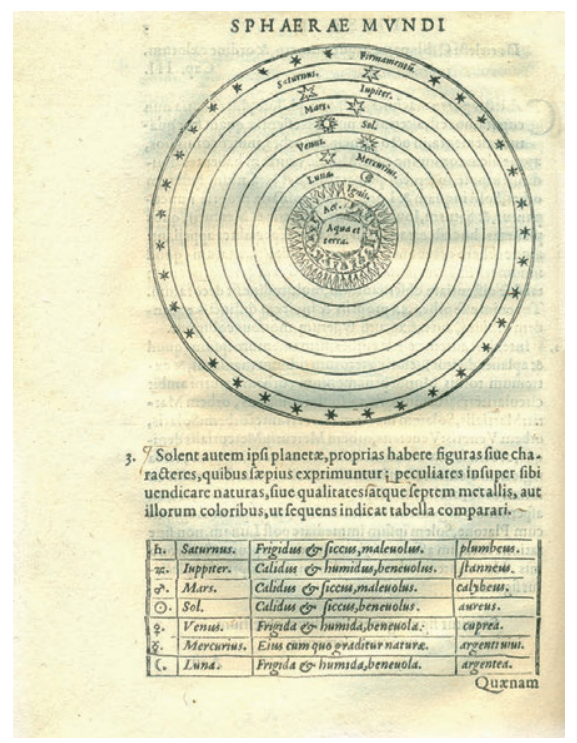

Fig. 5 - ORONZIO FINEO IDEM SPHAERAE MUNDI LIBER I CAP. III.

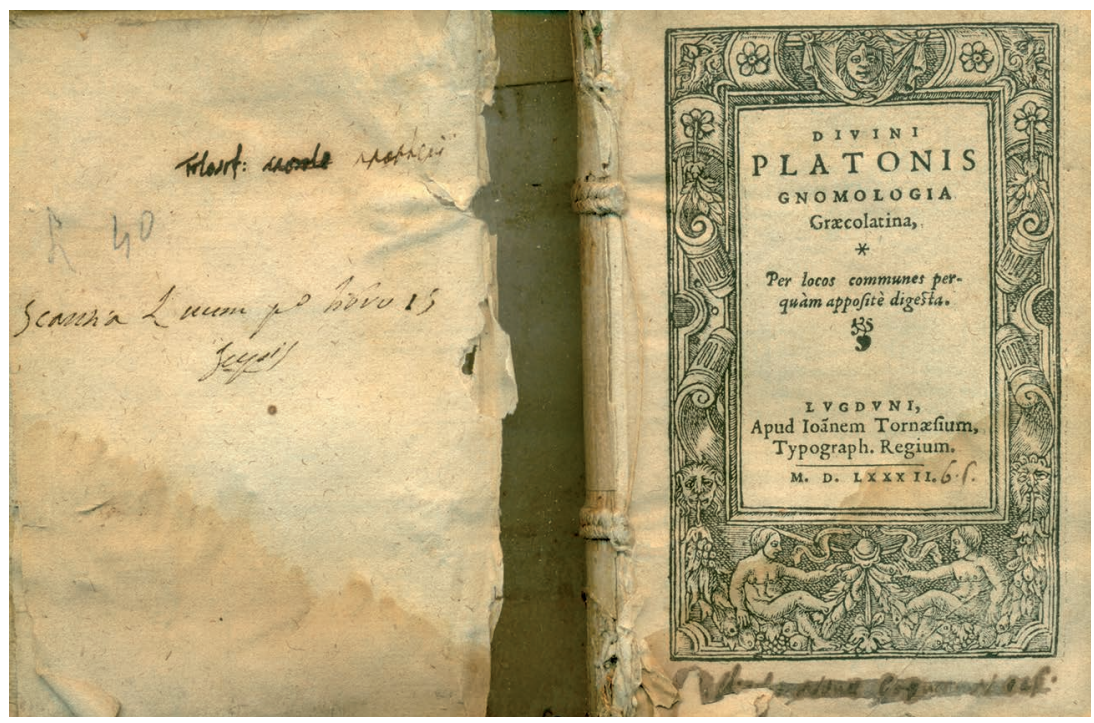

Fig. 6 - PLATONE (Atene 428 a.C.-348 a.C.) GNOMOLOGIA PLATONIS Apud Ioinem Tornæsium -Lugduni $1582 \mathrm{~b} .11 .5 \mathrm{~cm}$. 


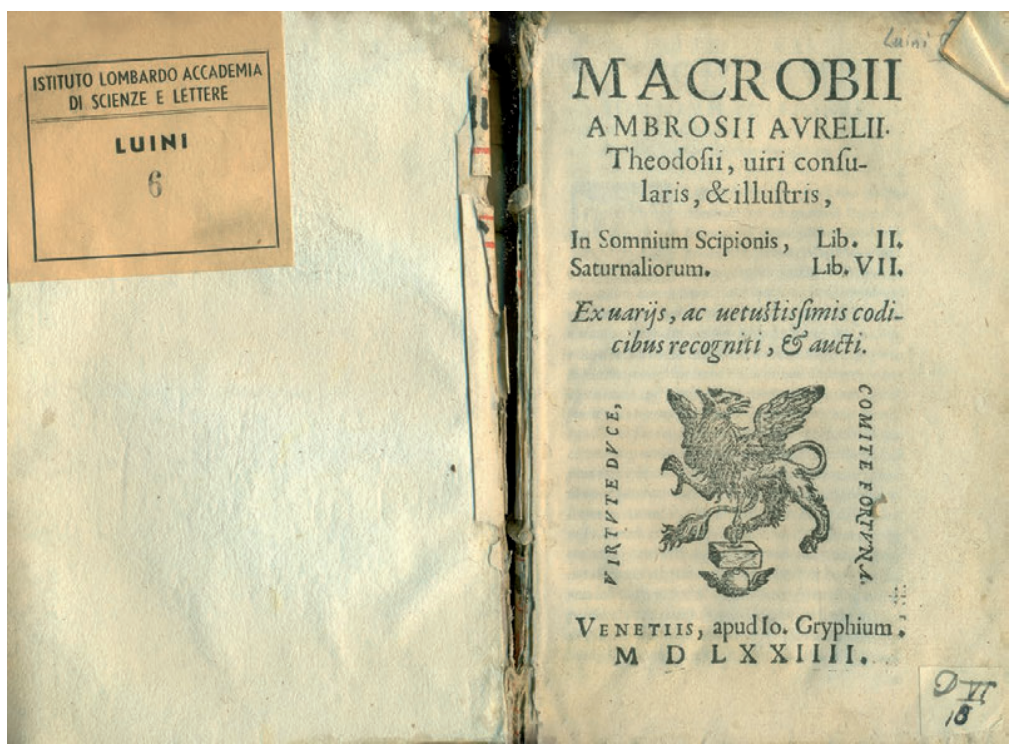

Fig. 7 - MACROBII AMBROSII (390-430 circa) In Somnium Scipionis lib. II Saturnaliorum lib. VII Venetiis, ap.Gryphium 1574 h. 15,5 cm.

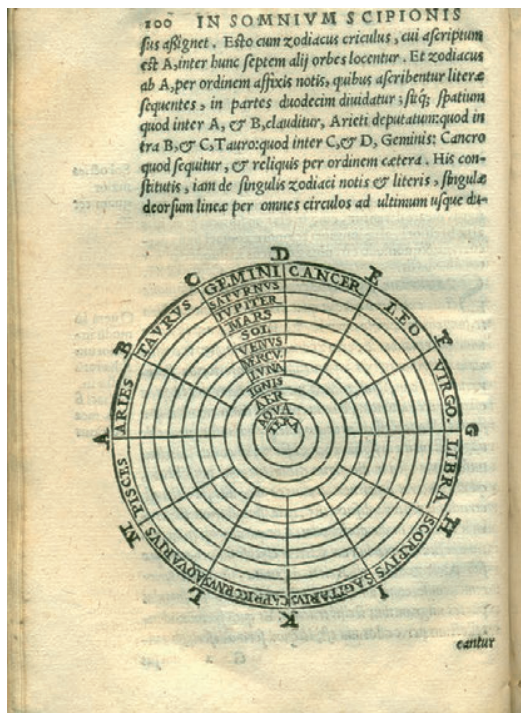

TI L I B R I. cantur: proculdabio per orbes fingulos dicodencs partes dusidet tranfius linedrian. In quocungue igitur circtlo fou

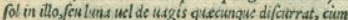

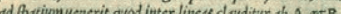

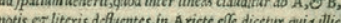

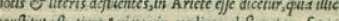
confl tuta fostion A rictes in zodiaco defignatiom fuper uerticem, ficut deforipfinus, hubebit. Similiter in quaicuen;

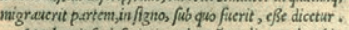
Atg; bec ipfa deforiptio eodem copendio nos docebit, Cur alix

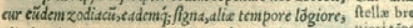

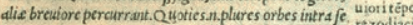

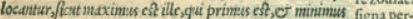
qui locum ultimuin tenctitus de medij ssqui finmo propior curntht,

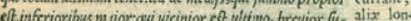

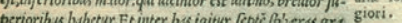
dis ecleritus fue fugutis ordo pofitionis aferipfit. I deoftel

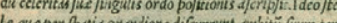

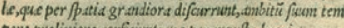

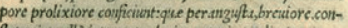
fat enim nelli inter est celerins ceteris tindiu' ue proce

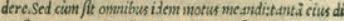

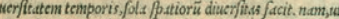

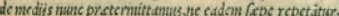

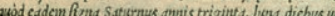
gintiofo on er ran anien /ungulespro/pstif fiu modostepus nieandi aut ext dest, axt contrahunt. Hoc loco diligens rerum difauf. Quomofor invertict, quod requirat . infpettis enim zodiaci notis, do zodiaqus möftrat is prefitium fidei adicocata defcriptio. Q uis cus induo ukró,inguist, circi coxlostis ditodecin partes aut inuenit, decim par

$$
\text { G } 3 \text { ait fis. }
$$

Fig. 8 - IDEM Liber I ZODIACO pagg.100-101. 


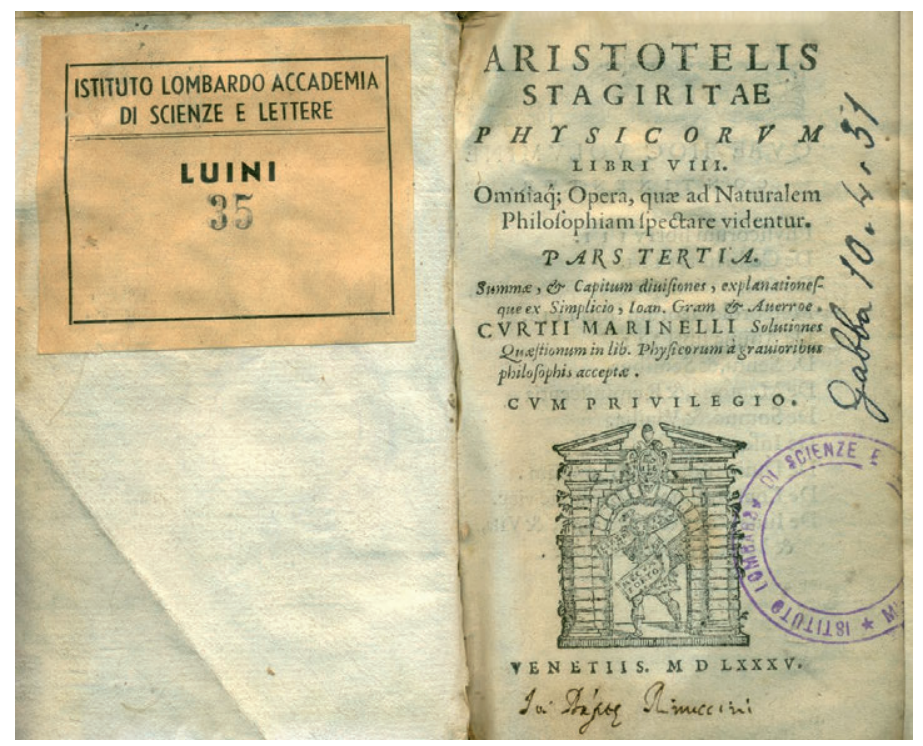

Fig. 9 - CURTII MARINELLI (Nardi 1476-1563) ARISTOTELIS STAGIRITÆ PHYSICORUM LIBER VIII PARS TERTIA VENETIIS 1585 h. 11.5 .

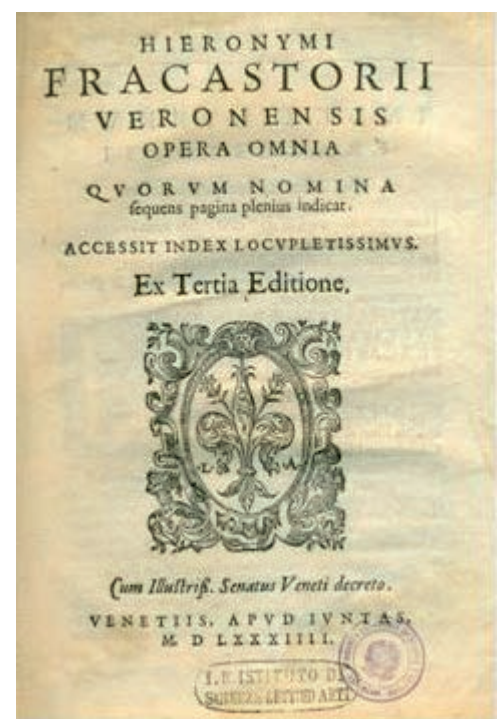

Fig. 10 - HIERONYMI FRACASTORII (Verona 1476-Affi 1553) OPERA OMNIA VENETIIS AP. IUNTAS 1584. 


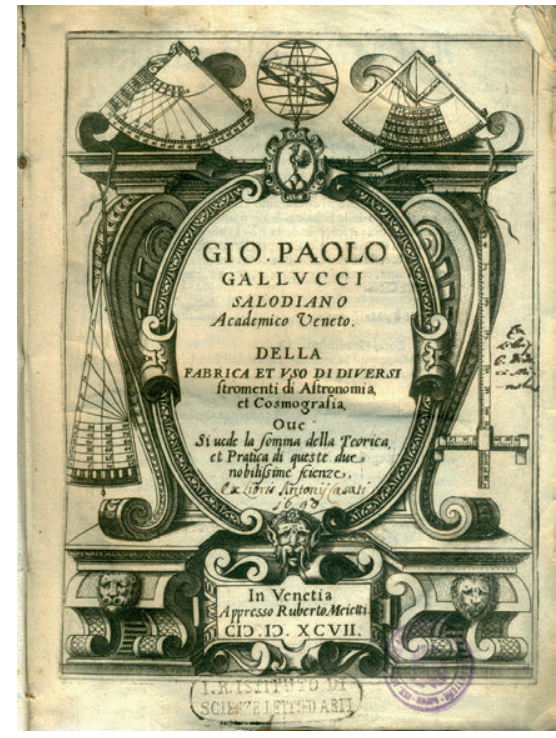

Fig. 11 - Frontespizio.

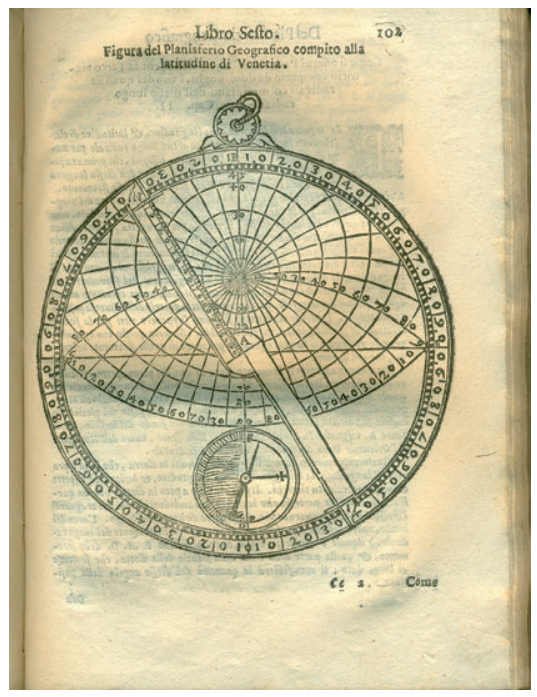

Fig. 12 - Planisfero.

GIO.PAOLO GALLUCCI (Salò 1538-Venezia 1621) DELLA FABRICA ET USO DI DIVERSI Stromenti di Astronomia et Cosmografia, In Venetia Appresso Ruberto Meietti 1597 h. 21,5. 


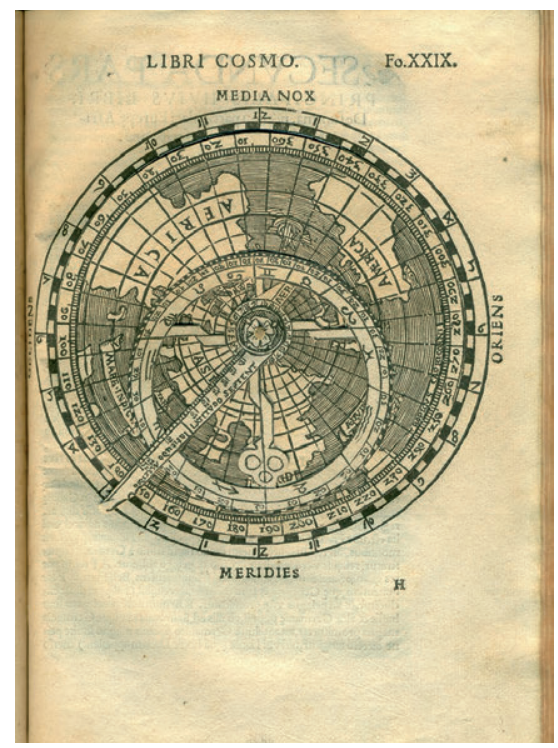

Fig. 13 - Uso planisfero.

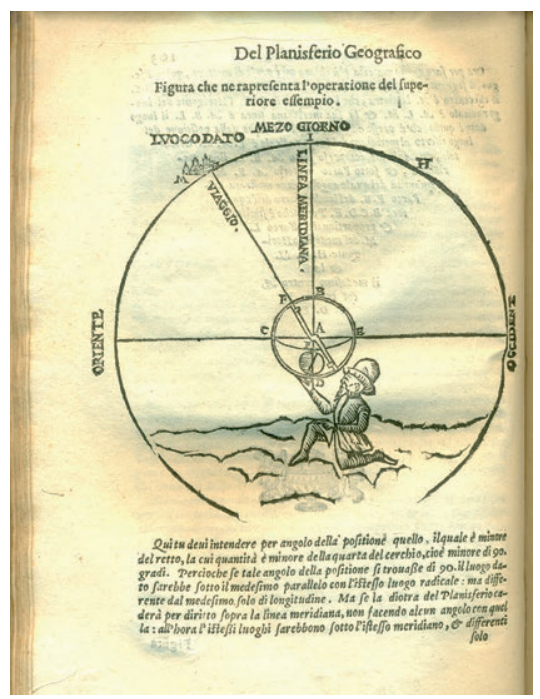

Fig. 14 - PLANISFERO GEOGRAFICO CON MAPPA DEI CONTINENTI.

GIO.PAOLO GALLUCCI (Salò 1538-Venezia 1621) DELLA FABRICA ET USO DI DIVERSI Stromenti di Astronomia et Cosmografia LUINI159

In Venetia Appresso Ruberto Meietti 1597 h. 21,5. 


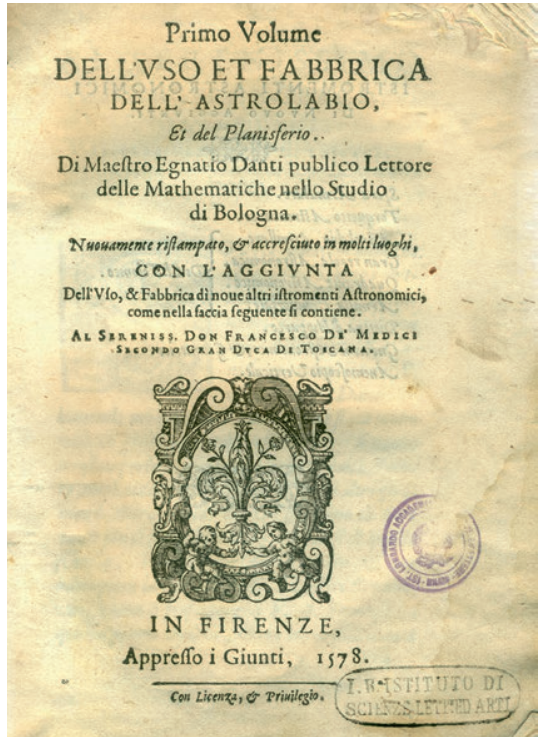

Fig. 15 - Frontespizio.

VSO DELLASTAOIABTO. codel Capricorno fino al punto nella parte di Ponente fegna to G. \& tirafi fra li due punti vna linea occulta, e ponendo il compaffo nel centro $E$. defcriue il ctrcolo Equinorziale, chic pasfià punto doue la lineagia detta interfeca la linea Meridiana nel punto K. Dinuouo tirifi vn'alera linea occulta dal pí to, dech to, dice for chio, chefaràil Tropico del Cancro.

EQVINOZZIALE, ET TROPICI.

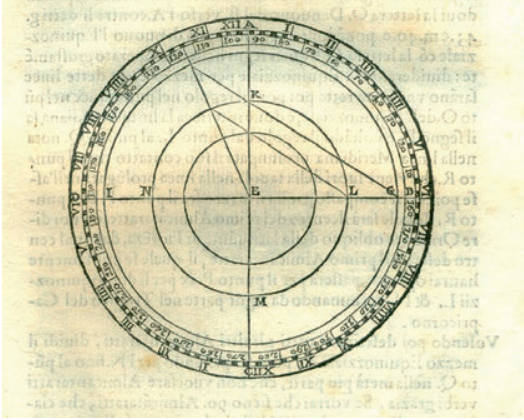

Fig. 17 - Uso astrolabio.

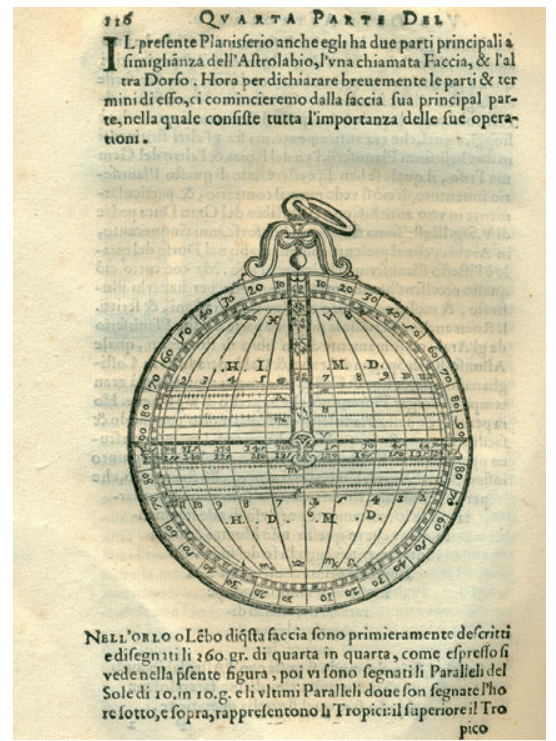

Fig. 16 - Planisfero.

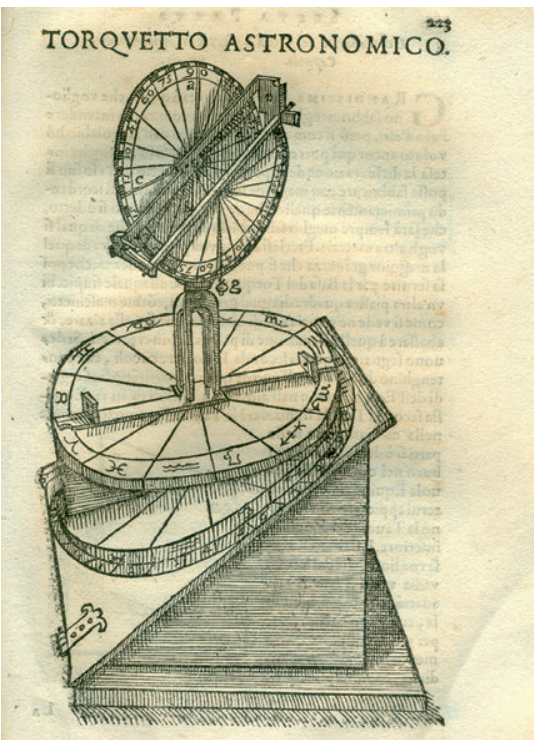

Fig. 18 - Torquetto.

EGNATIO DANTI Dell'uso e fabbrica dell'ASTROLABIO in FIRENZE Appresso $i$ Giunti, 1578 h. 21,5 cm. 


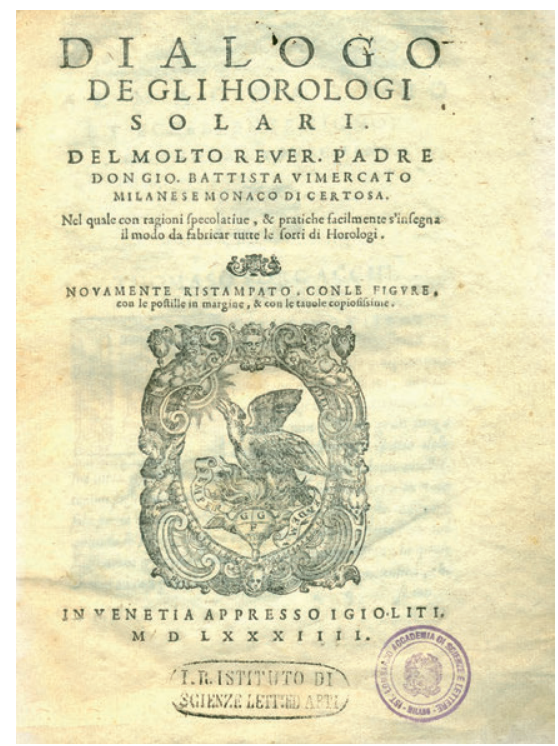

Fig. 19 - Frontespizio.

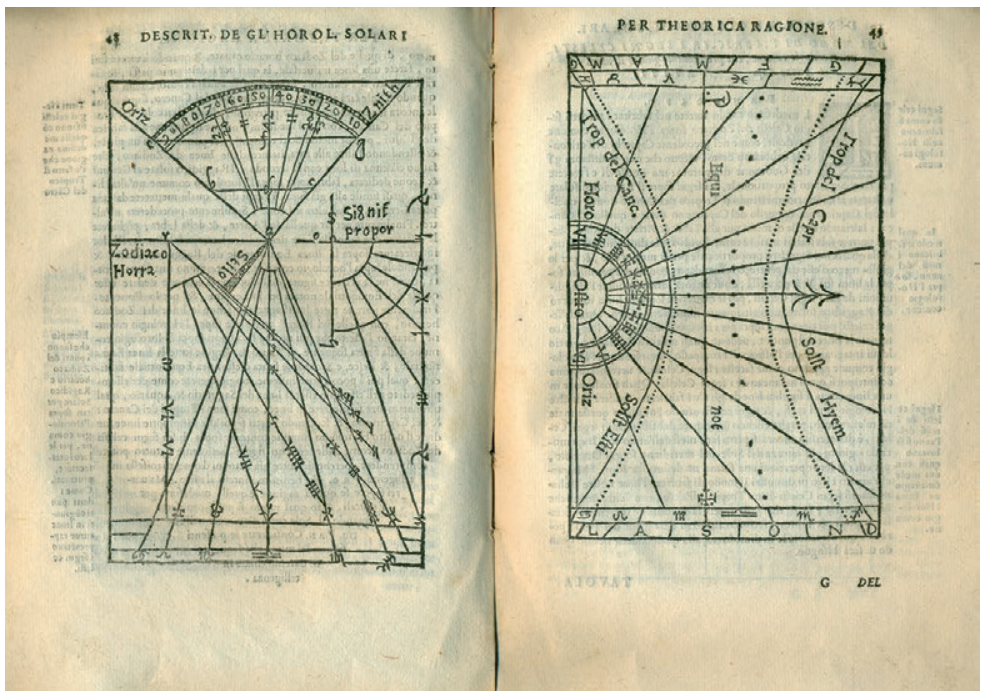

Fig. 20 - Meridiana.

GIO.BATTISTA VIMERCATO DIALOGO DEGLIOROLOGI SOLARI b. 21,5 cm. In VENETIA APPRESSO IGIOLITI 1584. 


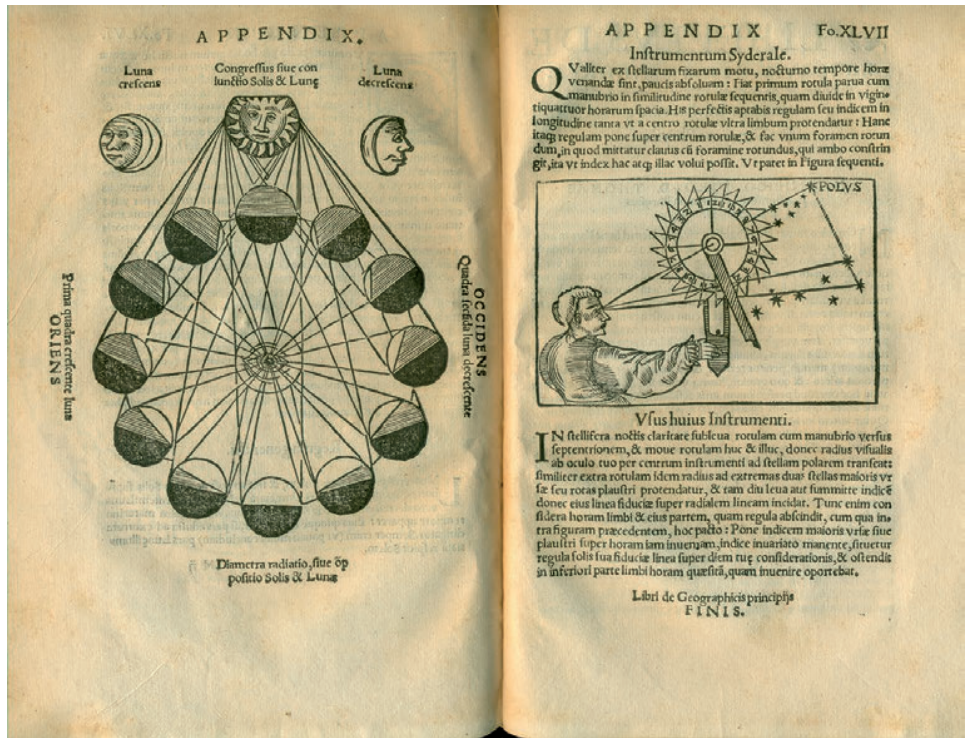

Fig. 21 - Pietro Apiano Instrumentum Syderale Væxeunt Antuerpix in pingui gallina Arnoldo Berckmãno 1539. b. 23,7 cm.

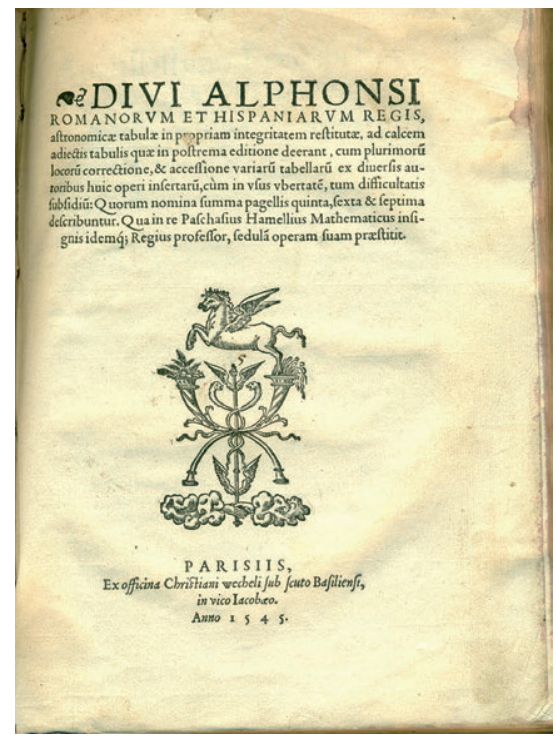

Fig. 22 - Alfonsine Pariis (1252) 1545. 


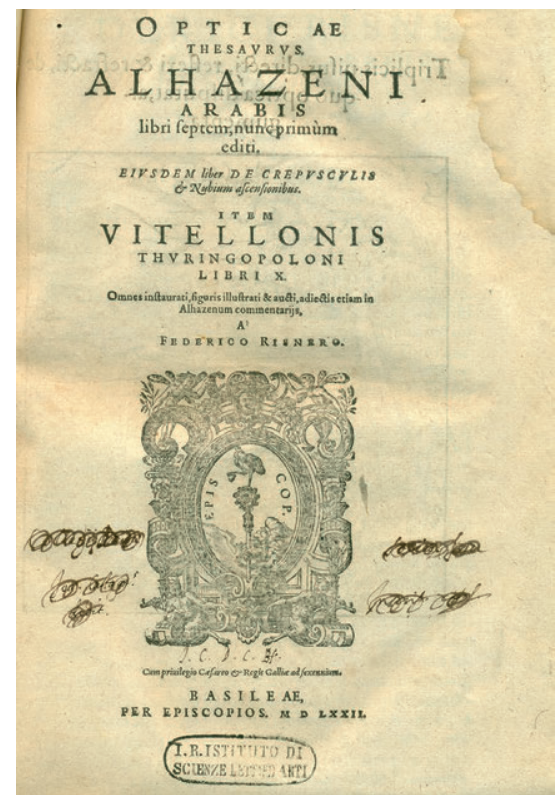

Fig. 23 - Frontespizio.

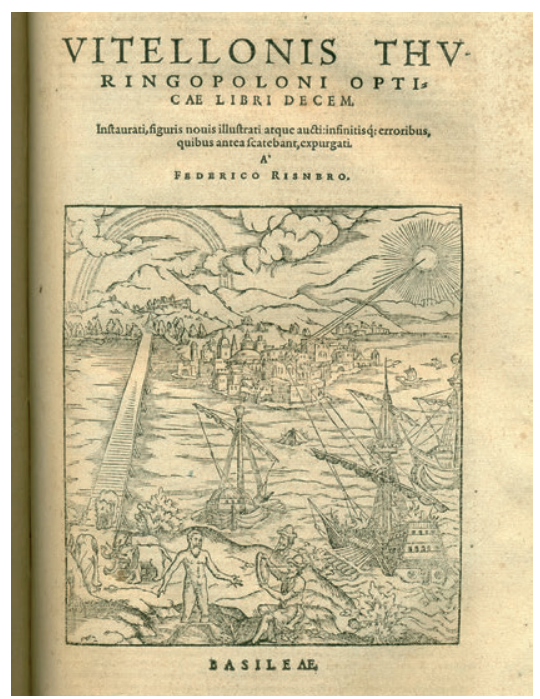

Fig. 24 - Figura interna.

ALHAZENI \& VITELLONIS - OPTICAE THESAURUS BASILEÆ PER EPISCOPIOS, $1572 \mathrm{~h} .33 \mathrm{~cm}$. 